\title{
Density Measurement of 1-D Confined Water by Small Angle Neutron Scattering Method: Pore Size and Hydration Level Dependences
}

\author{
Dazhi Liu, ${ }^{\dagger}$ Yang Zhang, ${ }^{\dagger}$ Yun Liu,,$+\perp$ Jianlan Wu, ${ }^{\dagger}$ Chia-Cheng Chen, ${ }^{\S}$ \\ Chung-Yuan Mou, ${ }^{\S}$ and Sow-Hsin Chen*, ${ }^{\dagger}$ \\ Department of Nuclear Science and Engineering, Massachusetts Institute of Technology, Cambridge, \\ Massachusetts 02139, NIST Center of Neutron Research, National Institute of Standard and Technology, \\ Gaithersburg, Maryland, Department of Materials Science and Engineering, University of Maryland, College \\ Park, Maryland, and Department of Chemistry, National Taiwan University, Taipei, Taiwan 106
}

Received: October 25, 2007; In Final Form: January 27, 2008

\begin{abstract}
Small angle neutron scattering (SANS) is used to measure the absolute density of water contained in 1-D cylindrical pores of a silica material MCM-41-S with pore diameters of 19 and $15 \AA$. By being able to suppress the homogeneous nucleation process inside the narrow pore, one can keep water in the liquid state down to at least $160 \mathrm{~K}$. From a combined analysis of SANS data from both $\mathrm{H}_{2} \mathrm{O}$ and $\mathrm{D}_{2} \mathrm{O}$ hydrated samples, we determined the absolute value of the density of 1-D confined water. We found that the average density of water inside the fully hydrated $19 \AA$ pore is $8 \%$ higher than that of the bulk water at room temperature. The temperature derivative of the density shows a pronounced peak at $T_{\mathrm{L}}=235 \mathrm{~K}$ signaling the crossing of the Widom line at ambient pressure and confirming the existence of a liquid-liquid phase transition at an elevated pressure. Pore size and hydration level dependences of the density are also studied.
\end{abstract}

Among many thermodynamic anomalies of water, the most notable one is the existence of the density maximum at $277 \mathrm{~K}$ and the minimum at $203 \mathrm{~K} .{ }^{1,2}$ The presence of these density extrema imply the existence of the hypothetical liquid-liquid critical point (LLCP) hidden in the deeply supercooled temperature in-between these two extrema at an elevated pressure. ${ }^{3}$ Recent neutron scattering experiments ${ }^{4}$ and molecular dynamics (MD) simulations ${ }^{5}$ render further support of the existence of the LLCP. A reliable determination of the equation of state, such as the density as functions of temperature and pressure, is thus essential in verifying the location of the LLCP in the phase diagram. Scientists have been studying the density of supercooled water since 1837. ${ }^{6}$ In 1987, Hare and Sorensen measured the density of supercooled water at its homogeneous nucleation temperature $(\sim 239 \mathrm{~K}),{ }^{7}$ the limiting temperature below which bulk water cannot remain in the liquid state. A confining substrate, such as nanoporous silica material MCM-41, can be used to inhibit the crystallization process and the confined water can be supercooled down to at least $160 \mathrm{~K}$. This trick allows us to study the relaxational behavior of water in this "no-man's land". ${ }^{4}$

Besides its importance to fundamental physics of water, confined water exhibits many novel liquid properties found in living cells. ${ }^{8}$ Density of water near a hydrophilic or a hydrophobic interface reflects the special hydrogen-bond configuration near the confining surface of the substrate. For example, protein hydration water has been shown ${ }^{9}$ to have the similar fragileto-strong dynamic crossover (FSC) as the confined water in MCM-41. ${ }^{4}$ The FSC marks the crossover of the local structure of water from predominantly a high-density liquid (HDL) to a low-density liquid (LDL). The former (HDL) is in a more fluid

\footnotetext{
* To whom correspondence should be addressed. E-mail: sowhsin@
} mit.edu.

$\dagger$ Massachusetts Institute of Technology.

$\doteqdot$ National Institute of Standard and Technology.

$\S$ National Taiwan University.

${ }^{\perp}$ University of Maryland. state while the latter (LDL) is in a less fluid state. ${ }^{9,10}$ This change of hydration water mobility at the crossover may trigger the so-called glass transition in protein. ${ }^{11}$

Small angle neutron scattering method can be used to measure the average density of water in the pore. The reason is that the neutron scattering intensity is proportional to the square of the difference of the scattering length density (sld) between the confined liquid and the substrate. The sld of a molecular liquid is equal to the number density of the liquid times the scattering length of the molecule. Since the scattering length of a nucleus does not change with temperature, the temperature variation of the sld is directly proportional to the variation of the number density (or the mass density) of the liquid. In 1993, the relative densities of water and ice were measured in porous solids and a montmorillonite clay. ${ }^{12}$ In the case of water confined in MCM41 , due to the 2-D hexagonal structure of the silica matrix, the scattering intensity distribution displays a peak. The height of this diffraction peak reflects the square of the mass density of the confined liquid since the density of silica has a very weak temperature dependence in the temperature range studied. We used $\mathrm{D}_{2} \mathrm{O}$ instead of $\mathrm{H}_{2} \mathrm{O}$ to do the density measurement since the coherent sld of $\mathrm{D}_{2} \mathrm{O}$ is much larger than that of $\mathrm{H}_{2} \mathrm{O}$. Hence it is easy to see the temperature dependence of the density of $\mathrm{D}_{2} \mathrm{O}$ through the diffraction peak height. Using this method, we have shown recently that water has a density minimum in the supercooled region which has never been found before. ${ }^{2}$ In this article, we improved the method of density measurement further and developed a new way to determine the absolute density of 1-D confined $\mathrm{D}_{2} \mathrm{O}$ by simultaneously taking the SANS data of $\mathrm{D}_{2} \mathrm{O}$ and $\mathrm{H}_{2} \mathrm{O}$.

The measured water density shows a smooth transition from a high-density level to a low-density level, which was predicted by MD simulations. ${ }^{5,13,14}$ Combining the experimental results of the transition between the low density (LDA) and the high density amorphous solid water (HDA), ${ }^{15,16}$ a complete picture of water in the low-temperature region emerges. Although more 
data are needed to map out the temperature dependence of density along many high pressure isobars, in this paper we demonstrate that in principle this method is capable of locating the LLCP if it exists.

Using this new method we found that the average density of $\mathrm{D}_{2} \mathrm{O}$ confined in the fully hydrated MCM-41-S-19 is $8 \%$ higher than that of the bulk $\mathrm{D}_{2} \mathrm{O}$ at room temperature. This result is supported by MD simulations ${ }^{17,18}$ and by a direct measurement of the density of the hydration water on protein surface. ${ }^{19}$

SANS experiments were performed at NG7, a 30-m SANS spectrometer, in the NIST Center for Neutron Research (NCNR). The incident neutrons have an average wave length $\lambda=5 \AA$ with fractional wavelength spread of $\Delta \lambda / \lambda=10 \%$. Sample to detector distance was fixed at 6 meters, covering the magnitude of the neutron wave-vector transfer $\mathrm{Q}$ range from 0.008 to 0.35 $\AA^{-1}$. This $\mathrm{Q}$ range covers the first Bragg peak (due to the hexagonal array of silica pores) which is used as an indicator of the density of water in the sample.

The sample consists of MCM-41-S powder, which is made of a micellar templated mesoporous silica matrix and has 1-D cylindrical pores arranged in a 2-D hexagonal lattice., ${ }^{40}$ Synthesis and characterizations of MCM-41-S were described in ref 20 in detail.

The sample is hydrated by exposing it to water vapor $\left(\mathrm{D}_{2} \mathrm{O}\right.$ or $\left.\mathrm{H}_{2} \mathrm{O}\right)$ in a closed chamber until it reaches the fully hydration level (approximately $0.5 \mathrm{~g}$ of $\mathrm{D}_{2} \mathrm{O} / 1 \mathrm{~g}$ of MCM-41-S) at room temperature for the $19 \AA$ sample. We controlled the hydration process of the $15 \AA$ sample to reach $85 \%$ of its fully hydration level. We made measurements at a series of temperatures between 140 and $290 \mathrm{~K}$ in a step of $10 \mathrm{~K}$ (or $5 \mathrm{~K}$ ) to monitor the variation of the density in the supercooled and at the lowtemperature region above the melting point for the $\mathrm{D}_{2} \mathrm{O}$ hydrated sample. The $\mathrm{H}_{2} \mathrm{O}$ sample was measured at two temperatures, 160 and $260 \mathrm{~K}$, to make the calibration.

In neutron scattering experiments, the scattering intensity distribution reflects the 3-D Fourier transform of the spatial structure of the sample. A peak in the scattering intensity distribution reveals a periodical structure of the scattered center in the real space. The powder sample of MCM-41-S consists of a collection of micron size grains and in each grain a bundle of cylindrical pores arranged in a 2-D hexagonal lattice. Figure 1 shows SANS peaks of the $19 \AA$ sample situated at $Q_{1}=0.19$ $\AA^{-1}$, which is related to the center-center distance $a$ of the cylindrical pores in the hexagonal structure by $a=2 \pi / Q_{1} \approx$ $33 \AA$ A.

SANS intensity distribution $I(Q)$ of an isotropic sample is given by $I(Q)=n V_{\mathrm{p}}^{2}(\Delta \rho)^{2} \bar{P}(Q) S(Q)$ after the interfacial scattering between grains has been subtracted from the signal. $n$ is the number density of the particle or the scattering unit (the 1-D cylindrical column in this case), $V_{\mathrm{p}}$ is the volume of the scattering unit, $\Delta \rho=\rho_{\mathrm{p}}-\rho_{\mathrm{e}}$ is the difference of the scattering length density (sld) between the water column $\rho_{\mathrm{p}}$ and the silica matrix $\rho_{\mathrm{e}}, \bar{P}(Q)$ is the normalized particle structure factor of the scattering unit, and $S(Q)$ is the inter-particle structure factor. The sld $\rho_{\mathrm{p}}$ can be rewritten as $\rho_{\mathrm{p}}=\alpha \rho_{\mathrm{p}}^{m}$, where $\alpha=\left(N_{\mathrm{A}} \sum_{i} b_{i}\right) / M_{\mathrm{w}}, N_{\mathrm{A}}$ is Avogadro's number, $M_{\mathrm{w}}$ is the molecular weight of water, $b_{i}$ is the coherent scattering length of the $i$ th atom in the scattering unit, and $\rho_{\mathrm{p}}^{m}$ is the average mass density of the water column. The sld of the silica matrix $\rho_{\mathrm{e}}$ can be determined by a contrast variation experiment. ${ }^{21} \mathrm{~A}$ contrast variation experiment was performed and showed that the match point corresponds to the molar ratio of $\left[\mathrm{D}_{2} \mathrm{O}\right]:\left[\mathrm{H}_{2} \mathrm{O}\right]$ $=0.66: 0.34$. Therefore, the sld $\rho_{\mathrm{e}}$ of the confining material

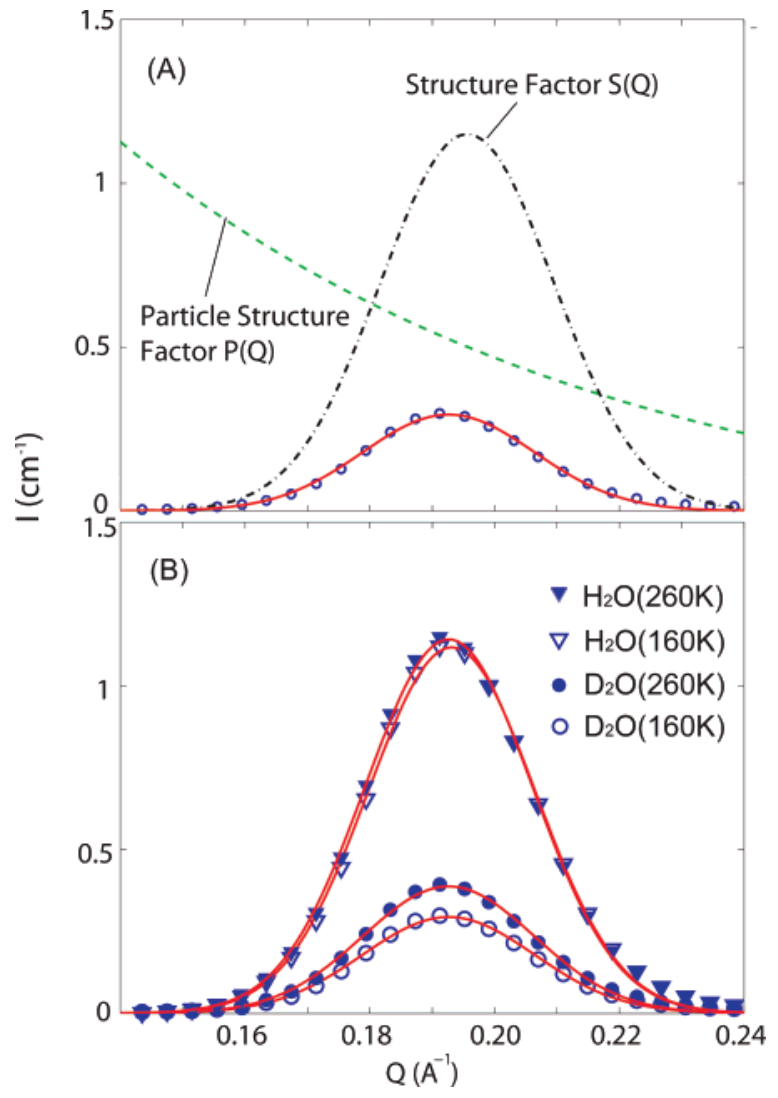

Figure 1. Model analysis of SANS intensity distribution of fully hydrated sample in $19 \AA$ pores. (A) The open circles show the SANS data. The solid line represents the fitted curve using the model given in the text. The dash-dotted line represents the structure factor of the 2-D hexagonal lattice (the third factor in eq 2). The dashed line represents the particle structure factor of the cylindrical tube of $\mathrm{D}_{2} \mathrm{O}$ column (the second factor in eq 2). (B) Comparison of the spectrum of $\mathrm{D}_{2} \mathrm{O}$ hydrated sample and $\mathrm{H}_{2} \mathrm{O}$ hydrated sample at two temperatures. The scattering intensities of $\mathrm{D}_{2} \mathrm{O}$ hydrated sample are lower than which of $\mathrm{H}_{2} \mathrm{O}$ hydrated sample. But the intensity variation as a function of the temperature change is higher due to the relatively higher sld of $\mathrm{D}_{2} \mathrm{O}$ sample. This figure also shows good agreement between the experimental data (symbols) and the model fitted curves (solid lines).

MCM-41-S is $4.006 \times 10^{10} \mathrm{~cm}^{-2}$ which is independent of temperature in this temperature range.

The normalized particle structure factor $\bar{P}(Q)$ of a long $(Q L$ $>2 \pi)$ cylinder is $P(Q)=\pi / Q L\left(2 J_{1}(Q R) / Q R\right)^{2},{ }^{22}$ where $L$ and $R$ represent the length and the radium of the cylinder, respectively, and $J_{1}$ is the first-order Bessel function of the first kind. The structure factor $S(Q)$ of a perfect 2-D hexagonal lattice is a series of delta functions (Bragg peaks) situated at $Q_{0}=0, Q_{1}$ $=2 \pi / a, Q_{2}=\sqrt{ } 3(2 \pi / a) \ldots$, where $a$ is the length of the primitive vectors of the 2-D hexagonal lattice. Except for $Q_{0}$, all of the Bragg peaks will broaden due to defects of the lattice and can be well approximated by a Lorentzian function. Therefore, the neutron intensity we measured in the $Q_{1}$ peak region $(0.1-0.3$ $\AA^{-1}$ ) is expressed as

$I(Q)=$

$n V_{\mathrm{p}}^{2}\left(\alpha \rho_{\mathrm{p}}^{m}-\rho_{\mathrm{e}}\right)^{2} \frac{\pi}{Q L}\left(\frac{2 J_{1}(Q R)}{Q R}\right)^{2}\left(C_{0} \frac{\frac{1}{2} \Gamma}{\left(Q-\frac{2 \pi}{a}\right)^{2}+\left(\frac{1}{2} \Gamma\right)^{2}}\right)$

where $\Gamma$ is fwhm and $C_{0}$ is a temperature-independent constant. Combining all constants, we obtain 


$$
I(Q)=C \frac{J_{1}(Q R)^{2}}{Q^{3} R^{2}}\left(\frac{\frac{1}{2} \Gamma}{\left(Q-\frac{2 \pi}{a}\right)^{2}+\left(\frac{1}{2} \Gamma\right)^{2}}\right)
$$

where the new prefactor $C=G\left(\alpha \rho_{\mathrm{p}}^{m}-\rho_{\mathrm{e}}\right)^{2}$. Factor $G$ is defined as $G=4 \pi n C_{0} V_{\mathrm{p}}^{2} / L$, which is a temperature-independent constant and is only determined by the geometry of the sample. From the definition of $\alpha$, one can easily calculate that $\alpha_{\mathrm{H}_{2} \mathrm{O}}=$ $-0.5613 \times 10^{10} \mathrm{~cm} / \mathrm{g}$ and $\alpha_{\mathrm{D}_{2} \mathrm{O}}=5.759 \times 10^{10} \mathrm{~cm} / \mathrm{g}$. Factor $C$ can be obtained by fitting the intensity curve using formula 2.

To obtain the absolute density of water, there's only one unknown, factor $G$, that needs to be determined. Considering that the sample geometries are kept identical during different measurements, factor $G$ can be canceled out by combining two measurements. In our experiment, we measured the scattering intensity of the $\mathrm{D}_{2} \mathrm{O}$ hydrated sample and the $\mathrm{H}_{2} \mathrm{O}$ hydrated sample at 160 and $260 \mathrm{~K}$ (see Figure 1). Assuming at these temperatures $\rho_{\mathrm{D}_{2} \mathrm{O}}^{m}=1.1 \rho_{\mathrm{H}_{2} \mathrm{O}}^{m}$, we wrote

$$
\rho_{\mathrm{D}_{2} \mathrm{O}}^{m}=\frac{1+\beta}{\alpha_{\mathrm{D}_{2} \mathrm{O}} \beta+\alpha_{\mathrm{H}_{2} \mathrm{O}} / 1.1} \rho_{\mathrm{e}}
$$

where $\beta=\left[C_{\mathrm{H}_{2} \mathrm{O}} / C_{\mathrm{D}_{2} \mathrm{O}}\right]^{1 / 2}$. Using the density of $\mathrm{D}_{2} \mathrm{O}$ extracted at $260 \mathrm{~K}$, the factor $G$ is determined. Then one can calculate the absolute values of $\mathrm{D}_{2} \mathrm{O}$ density for the whole temperature range.

Figure 2A shows the average $\mathrm{D}_{2} \mathrm{O}$ densities in the 19 and 15 $\AA$ pores versus temperature. The plot shows a smooth transition of $\mathrm{D}_{2} \mathrm{O}$ density from a high level to a low level. A density minimum occurs at $210 \pm 5 \mathrm{~K}$, which coincides with our previous publication. ${ }^{2} \partial \rho /\left.\partial T\right|_{\mathrm{p}}$ as a function of $T$ can be extracted from the $\rho$ vs $T$ curve (see Figure 2B). This is a quantity which is proportional to the thermal expansion coefficient $\alpha_{p}=-1 /$ $\rho(\partial \rho / \partial T)_{\text {P. }}$ It shows a pronounced peak at $235 \pm 5 \mathrm{~K}$. Since it is known that the absolute value of the thermal expansion coefficient peaks at the Widom line above the critical point, ${ }^{23,24}$ this result reinforces the plausibility of the existences of the Widom line and the associated LLCP. Plots of different pore sizes show almost the same peak positions. Therefore, although the absolute density of water in nanopores is different from that of bulk water, its temperature derivative $\partial \rho /\left.\partial T\right|_{\mathrm{p}}$ is independent of the absolute value of the density. Our experiment shows that the peak position of this quantity is independent of pore size.

Our previous neutron scattering experiments and NMR selfdiffusion constant measurements show that the FSC temperature of $\mathrm{H}_{2} \mathrm{O}$ is at $T_{\mathrm{L}}\left(\mathrm{H}_{2} \mathrm{O}\right)=225 \pm 5 \mathrm{~K}$ at ambient pressure. ${ }^{4,10}$ The value $T_{\mathrm{L}}\left(\mathrm{D}_{2} \mathrm{O}\right)=235 \pm 5 \mathrm{~K}$ for the FSC temperature of $\mathrm{D}_{2} \mathrm{O}$ estimated in this paper is thus reasonable because of the wellknown fact that thermodynamic quantities of $\mathrm{D}_{2} \mathrm{O}$ usually have a shift of several degrees to the higher temperature side compared to that of $\mathrm{H}_{2} \mathrm{O}$. For example, the density maximum of $\mathrm{D}_{2} \mathrm{O}$ occurs at $11{ }^{\circ} \mathrm{C}$ at ambient pressure instead of $4{ }^{\circ} \mathrm{C}$ for $\mathrm{H}_{2} \mathrm{O}$. MD simulations predicted that when pressure goes higher approaching the critical point, the peak of $\partial \rho /\left.\partial T\right|_{p}$ becomes increasingly higher and eventually diverges at the LLCP. ${ }^{5}$ It is plausible that using the method described above, one can eventually locate the LLCP by monitoring the growth of the peak height of $\partial \rho /\left.\partial T\right|_{p}$.

In principle, the density of any liquid that is confined in an ordered structure can be measured using the method discussed above. The only requirement is that the sld of the confined material and the substrate have a significant difference. Another obvious fact is that the sld of the material should be large

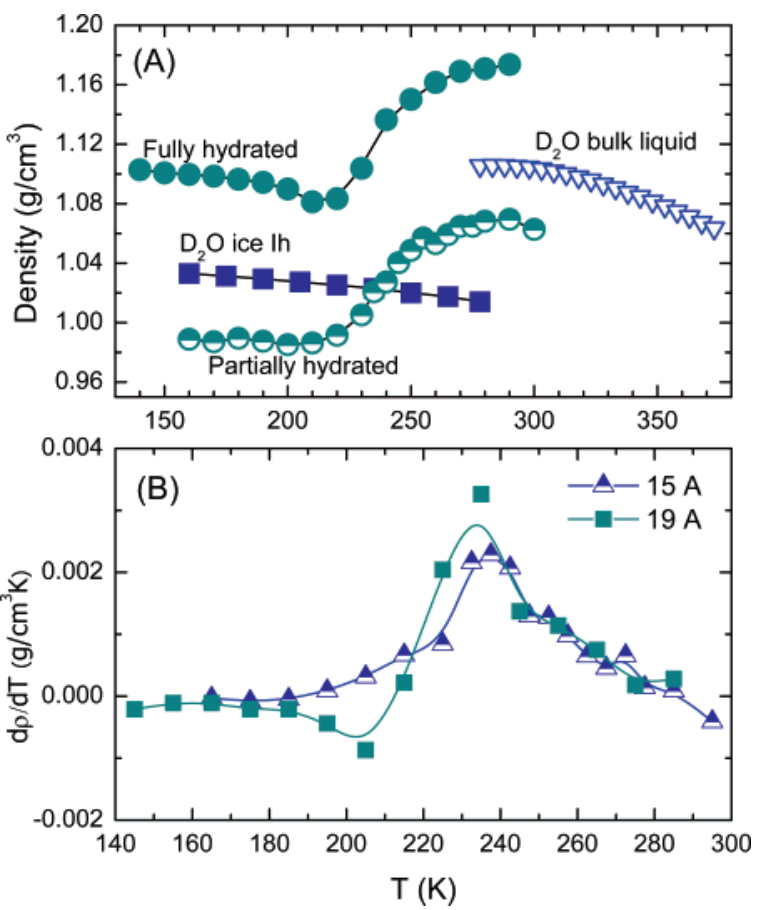

Figure 2. (A) Average $\mathrm{D}_{2} \mathrm{O}$ density inside the 19 and $15 \AA$ pores measured by SANS method as a function of temperature. The open triangular symbols are the density data for bulk $\mathrm{D}_{2} \mathrm{O}$ taken from CRC handbook..$^{26}$ It shows that the density of confined $\mathrm{D}_{2} \mathrm{O}$ in fully hydrated pores is $8 \%$ higher than bulk $\mathrm{D}_{2} \mathrm{O}$ at room temperature. $\mathrm{D}_{2} \mathrm{O}$ density in partially hydrated pores is lower than that of bulk water because of the existence of a partially empty central core. (B) The curve of $(\partial \rho /$ $\partial T)_{\mathrm{P}}$ vs T. (Density data were smoothed before calculating their derivatives.) Each curve shows a pronounced peak at $T_{\mathrm{L}}=235 \pm 5 \mathrm{~K}$, which is the temperature corresponding to the crossing of the Widom line ${ }^{24}$ at ambient pressure.

compared to that of the matrix to observe the density changes as a function of temperatures. Owing to a finite spatial resolution of the SANS method (about $10 \AA$ ), the measured density is the average density of the confined liquid in the pores. The accuracy of this measurement depends on the error bars of the SANS intensities around the peak position. From our analysis, our measured density has an error within $0.1 \%$, which means the results are reliable and do not sensitively depend on the fitting model.

This is the first experimental report of the measurement of the absolute density in 1-D confined water. At room temperature, our result shows that the density of confined water in a fully hydrated $19 \AA$ pore is $8 \%$ higher than that of the bulk water. Experiments and simulations show that there is a water shell close to the confining surface. The density of this layer of water is $10 \%$ to $15 \%$ higher than that of bulk water. ${ }^{17-19}$ The pore diameter of our sample is $19 \AA$ and the thickness of the surface layer is estimated to be $3 \AA$. From a simple calculation, we obtain the volume ratio of the central water to the shell water is about $4: 5$. Assuming the density of the shell water is $15 \%$ higher than that of the bulk water, we find the density of the central water is the same as that of the bulk water. To verify this result, we measured the water confined in partially hydrated pores of $15 \AA$ diameter with about $85 \%$ of the fully hydration level. Since water forms the shell layer first, ${ }^{25}$ the $15 \%$ lowering of hydration level is almost all taken from the central water (bulk water). The average density should drop about 5\% according to a similar calculation. The curves shown in Figure $2 \mathrm{~A}$ indicate a rough agreement of the numerical values of the measured density with this calculation. 
In conclusion, we give a method for an absolute density measurement of confined water. We also show the plausibility of the existence of the Widom line by demonstrating the peaking of the temperature derivative of density. In the previously published results, ${ }^{2}$ we studied the bulk water properties using the confined water assuming they have the same density at the room temperature. Although we verify in this paper that the interfacial layer of confined water (in MCM-41) has about $10 \sim 15 \%$ higher density than the bulk water, the main conclusion (of ref 2) of the existence of the density minimum is still valid.

Acknowledgment. The work is supported by the Department of Energy Grant DE-FG02-90ER45429. We profited from the affiliation with EU Marie-Curie Research and Training Network on Arrested Matter. We acknowledge the support of the Intense Pulsed Neutron Source, Argonne National Laboratory in terms of the allocation of the neutron beamtime for the preliminary experiments. We acknowledge the support of the National Institute of Standards and Technology, U.S. Department of Commerce, in providing the neutron research facilities used in this work.

\section{References and Notes}

(1) Mallamace, F.; Branca, C.; Broccio, M.; Corsaro, C.; Mou, C.-Y.; Chen, S.-H., Proc. Natl. Acad. Sci. 2007, 104, 18387-18391.

(2) Liu, D.; Zhang, Y.; Chen, C.-C.; Mou, C.-Y.; Poole, P. H.; Chen, S.-H. Proc. Natl. Acad. Sci. 2007, 104, 9570-9574.

(3) Poole, P. H.; Sciortino, F.; Essmann, U.; Stanley, H. E.; Nature 1992, 360, 324-328.

(4) Liu, L.; Chen, S.-H.; Faraone, A.; Yen, C.-W.; Mou, C.-Y. Phys. Rev. Lett. 2005, 95, 117802.

(5) Paschek, D. Phys. Rev. Lett. 2005, 94, 217802.

(6) Angell, C. A. Annu. Rev. Phys. Chem. 1987, 34, 593-630.

(7) Hare, D. E.; Sorensen, C. M. J. Chem. Phys. 1987, 87, 4840.
(8) Chaplin, M. Nat. Rev. 2006, 7, 861-866.

(9) Chen, S.-H.; Liu, L.; Fratini, E.; Baglioni, P.; Faraone, A.; Mamontov, E. Proc. Natl. Acad. Sci. 2006, 103, 9012.

(10) Mallamace, F.; Broccio, M.; Corsaro, C.; Faraone, A.; Wanderlingh,

U.; Liu, L.; Mou, C.-Y.; Chen, S.-H. J. Chem. Phys. 2006, 124, 161102.

(11) Tournier, A. L.; Xu, J.; Smith, J. C. Biophys. J. 2003, 85, 18711875.

(12) Li, J.-C.; Ross, D. K.; Heenan, R. K. Phys. Rev. B 1993, 48, 6716.

(13) Angell, C. A.; Bressel, R. D.; Hemmati, M.; Sare, E. J.; Tucker, J. C.; Phys. Chem. Chem. Phys. 2000, 2, 1559-1566

(14) Poole, P. H.; Saika-Voivod, I.; Sciortino, F. J. Phys. Condens. Matter 2005, 17, L431-L437

(15) Soper, A. K.; Ricci, M. A. Phys. Rev. Lett. 2000, 84, 2881-2884.

(16) Finney, J. L.; Hallbrucker, A.; Kohl, I.; Soper, A. K.; Bowron, D. T. Phys. Rev. Lett. 2002, 88, 225503.

(17) Merzel, F.; Smith, J. C. Proc. Natl. Acad. Sci. 2002, 99, 53785383.

(18) Lee, S. H.; Rossky, P. J. J. Chem. Phys. 1994, 100, 3334-3345.

(19) Svergun, D. I.; Richard, S.; Roch, M. H.; Sayers, Z.; Kuprin, S.; Zaccai, G. Proc. Natl. Acad. Sci. 1998, 95, 2267-2272.

(20) Liu, L.; Chen, S.-H.; Faraone, A.; Yen, C.-W.; Mou, C.-Y.; Kolesnikov, A.; Mamontov, E.; Leao, J. J. Phys. Condens. Matter 2006 $18, \mathrm{~S} 2261-\mathrm{S} 2284$.

(21) Edler, K. J.; Reynolds, P. A.; White, J. W. J. Phys. Chem. B 1998, $102,3676-3683$.

(22) Chen, S.-H.; Lin, T.-L.; Wu, C. F. In Physics of Amphiphilic Layers; Meunier, J., Langevin, D., Boccara, N., Eds.; Springer: Berlin, 1987; pp $241-252$

(23) Anisimov, M. A.; Sengers, J. V.; Levelt Sengers, J. M. Near-critical behavior of aqueous systems, Aqueous Systems at Elevated Temperatures and Pressures: Physical Chemistry in Water, Steam and Hydrothermal Solutions; Palmer, D. A., Fernandez-Prini, R., Harvey, A. H., Eds.; Amsterdam: Elsevier, 2004.

(24) Xu, L.; Kumar, P.; Buldyrev, S. V.; Chen, S.-H.; Poole, P. H.; Sciortino, F.; Stanley, H. E. Proc. Natl. Acad. Sci. U.S.A. 2005, 102, 1655816562

(25) Grunberg, B.; Emmler, T.; Gedat, E.; Shenderovich, I.; Findenegg, G. H.; Limbach, H.-H.; Buntkowsky, G. Chem. Eur. J. 2004, 10, 56895696.

(26) Lide, D. R., Ed.; CRC Handbook of Chemistry and Physics; Taylor and Francis: Boca Raton, FL, 2007; pp 6-8. 